\title{
Fracture monitoring by acoustic emission: recent applications of parameter-based characterization
}

\author{
D.G. AGGELIS*, J. BLOM*, S. DE SUTTER*, S. VERBRUGGEN*, M. \\ STRANTZA*, T. TYSMANS*, A. C. MPALASKAS $^{\dagger}$ AND P. L. NGUYEN ${ }^{\dagger \dagger}$ \\ "Vrije Universiteit Brussel \\ Brussels, Belgium \\ e-mail: daggelis@vub.ac.be \\ ${ }^{\dagger}$ University of Ioannina \\ Ioannina, Greece \\ ${ }^{\dagger \dagger}$ VK Vietnam \\ Ho Chi Minh City, Vietnam
}

\begin{abstract}
The present paper describes a collection of fracture monitoring cases in different materials. The cases examined include bending of textile reinforced cement (TRC), hybrid concrete-TRC lightweight beams, granite, additive manufacturing metal components, combined loading of human femur bone and pull-out in reinforced concrete. In all cases the basic role is played by acoustic emission (AE). It is shown that certain waveform parameters exhibit strong sensitivity to the rate of fracture as well as the dominant fracture mode. Parameters like frequency content and the duration of the signals supply real time trends that in the present cases are verified by optical techniques. It is concluded that $\mathrm{AE}$ supplies important information and allows the prediction of how the material will behave based on the initial AE recordings and before serious damage is inflicted. AE shows a very broad application range; however, the contribution of combination with other techniques is highlighted in order to increase the reliability of the interpretation of $\mathrm{AE}$ results.
\end{abstract}

\section{INTRODUCTION}

Acoustic emission (AE) is a technique unique in the sense that allows monitoring of processes even in the microscopic scale. In that respect it has proven very useful in detecting the existence and location of early damage in different materials, as well in characterizing the mode of fracture. Piezoelectric sensors are attached on the surface of the material being tested in order to record the transient elastic waves generated by cracking events. These sensors record the elastic waves on their surface in the form of electric waveforms [1]. A representation of an AE waveform is seen in Figure 1. Amplitude (A) is generally an important parameter as it correlates to the intensity of the fracture incident. Energy stands for the area under the rectified waveform. Additionally, the rise time (RT) is the delay between the first threshold crossing (or first count) and the peak amplitude. Rise time over amplitude (RA value) is also important, defined as RT divided by A and is measured by $\mu \mathrm{s} / \mathrm{V}$. Average frequency (AF) is the number of threshold crossings over the duration and it is a good approximation of the frequency content of the waveform. 


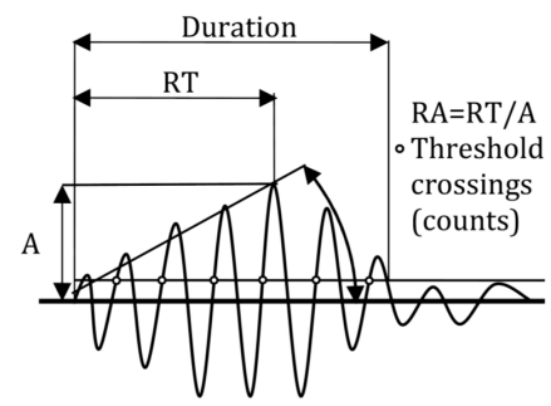

Fig. 1. Representation of an AE waveform with its basic parameters.

In $\mathrm{AE}$ literature one can see characterization approaches based on the full waveform (signal-based) and on the waveform parameters (parameter-based) [2,3]. The latter was applied the early years of $\mathrm{AE}$ as acquisition boards did not commonly have the capacity to record full waveforms. This is why in many cases these two approaches are mentioned as different but essentially they are complimentary. Most of contemporary equipment has the capacity to record full waveforms. This enables any treatment like extraction of features from frequency, wavelet domain or any other analysis that the user wishes. However, still the more traditional indices can be used if they supply the necessary information. Therefore, given the full waveform, it is each time the choice of the user to apply either simple parameters or analysis on the whole waveforms or both approaches. A few of the waveform parameters are of particular importance in this study as they have shown strong sensitivity on the fracture mode. These are the average frequency (AF) and the RA value (Rise time over amplitude). Additionally good trends are shown by rise time itself or duration of the signals. This paper discusses some recent cases of monitoring of material fracture, where the recorded parameters show strong sensitivity in the applied stress field and the ongoing fracture mechanisms. They are representative of the recent research in the Vrije Universiteit Brussel and indicative of the fields that AE parameter analysis can be used but they stand only for a small part of the applications in the field of AE worldwide. Each case is discussed separately and some general conclusions and common trends are stated later.

\section{MONITORING OF TEXTILE REINFORCED CEMENT (TRC) BEAMS}

TRC composites are a combination of Cement (in this case Inorganic Phosphate cement, IPC) with randomly distributed and oriented glass fibers. It is used for strengthening of concrete structures while it has good behaviour to high temperatures [4]. TRC is cast in thin and possibly curved-shaped structures. This makes prediction of its mechanical behavior a difficult task considering also the laminated, fibrous nature of the material. The fracture of this material includes matrix cracking, delaminations and fiber pull out. In the present case, thin beams of this material were fractured in three-point bending with different spans. While the shear stresses are nominally independent of the span, for longer spans, the normal stresses increase. Therefore, the stress field (or proportion between shear and tension) is strongly modified promoting different fracture mechanisms (matrix cracks or delaminations) [5]. It should be clear that all aforementioned mechanisms are present in any TRC fracture experiment; however, their relative dominance may be shifted in favour of one or the other based on the applied stress field. As an example for short span specimens, fracture phenomena related to the interlaminar shear stresses (delaminations) are expected to be more frequent than for high aspect ratios, which resemble more the pure tension loading introducing mostly normal stresses. This fracture behavior is reflected in the AE behavior in a quite clear manner, as explained below.

Fig. 2 shows an overview of the experiment and a photograph during test. The sensors were arranged in a straight line with increasing distance from the fracture zone in order to check the influence of propagation distance but in this manuscript we occupy only with the activity received by the two central sensors. They were broadband with peak sensitivity at $450 \mathrm{kHz}$. More details can be found in [5]. The important outcome of this monitoring is that AE can well follow the shift of the dominant fracture modes. In all cases, the initial deterioration is inflicted by cement cracking, which gives the necessary stress concentration in order for delaminations and pull-out to occur later. This is depicted in Fig. 3a where the moving average line of AF is depicted vs. time of loading for a "long" (aspect ratio of 83) and a "short" (aspect ratio of 15) span loading. It has been calculated by FEM [5] that for the long span the ratio of maximum shear to maximum tensile stress in the specimen is 0.03 while for 
the short this becomes 5 times higher. Both of them start with quite high values of frequency but later the frequency drops. While for the long specimen this happens nearly at the end of loading, for the short span, this drop in $\mathrm{AE}$ frequency comes much earlier (even within the first minute) showing the promotion of the shear-like damage mechanisms by the different stress field.

(a)
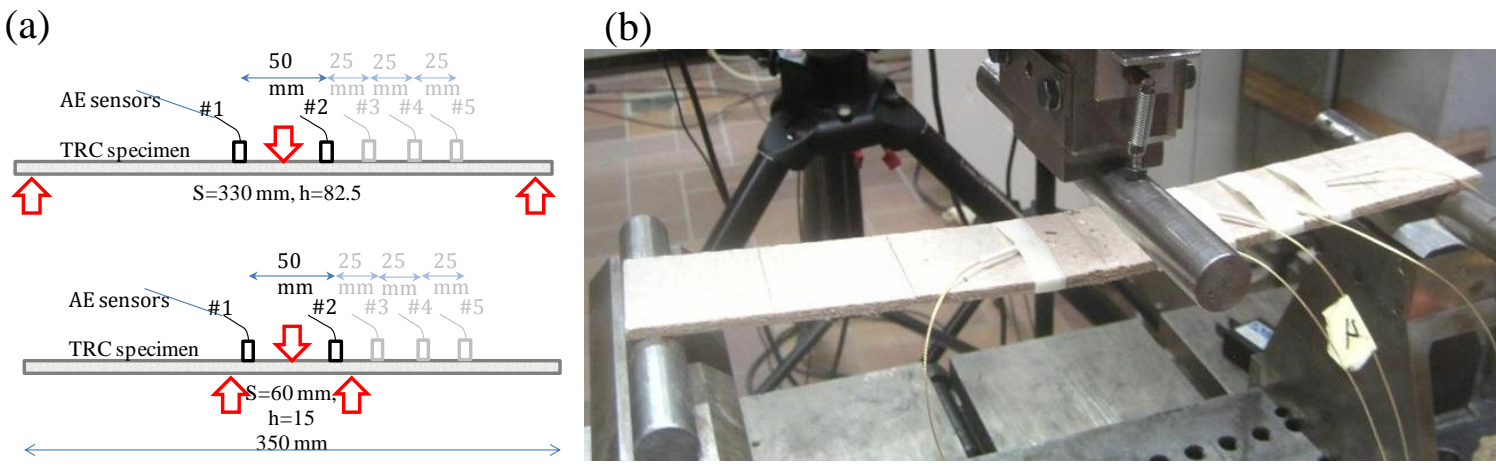

Fig. 2 (a) Representation of the bending set up and AE sensor locations, (b) photograph during the test.

Another very important aspect of the AE capabilities is that the differences are visible from the initial population of $\mathrm{AE}$, while the load is low and the structural condition has not yet been compromised. Fig. $3 \mathrm{~b}$ shows the average value of AF of the first 300 hits. This population in all cases was received at loads well below $20 \%$ of the ultimate. It is obvious that the frequency content of the emissions drops as the span is shortened (shear/normal 0.15) which reflects the changes in the preliminary manifestation of the fracture mechanisms. Therefore, in that sense it can be argued that AE is sensitive not only to the damage but also to the stress field that is responsible for the damage that will be developed. This sensitivity allows to provide feedback to the designers of complex shapes about the developed stresses and the expected mode of fracture just by applying a low level proof loading.

(a)

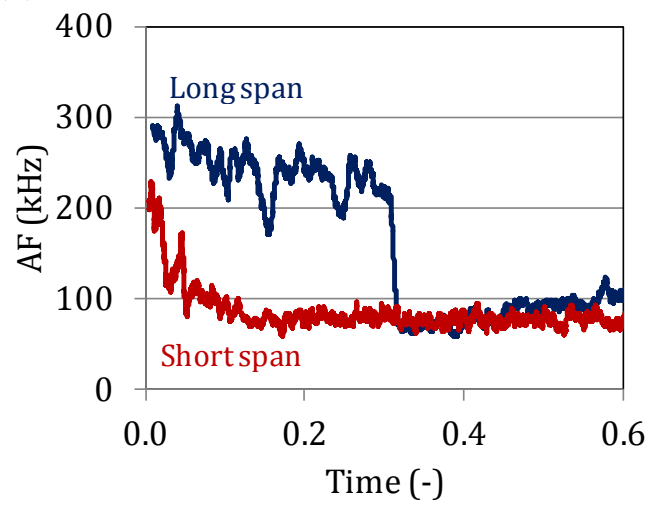

(b)

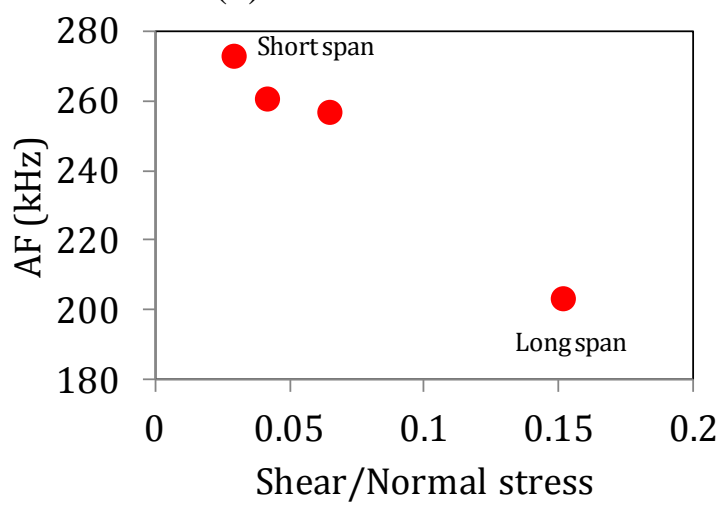

Fig. 3 (a) Average frequency (AF) trend line (moving average) for the long and the short spans. (b) Average value of AF of the first 300 hits received for the different spans.

\section{MONITORING OF HYBRID TRC-CONCRETE BEAMS DURING BENDING}

In this case, hollow rectangular beams of the aforementioned IPC-TRC material internally reinforced with carbon fiber reinforced polymer strips (CFRP) are combined with traditional concrete beams in a new hybrid beam design (see cross section of Fig. 4a). The aim is to form the structural element to support a lightweight floor system [6] and hence their structural and failure behaviour should be well understood. The mechanical test was four-point bending with span of $3 \mathrm{~m}$ and distance between loading points of $0.5 \mathrm{~m}$. The loading was monotonic, under displacement control until failure. Four AE sensors (resonant at $150 \mathrm{kHz}$ ) were attached on the concrete layer, as seen in Fig. 4b [7].

The mechanical behaviour and AE activity of this design was compared with similar beams lacking the CFRP reinforcement. The CFRP certainly increases the equivalent stiffness and the ultimate bearing capacity and alters the fracture mechanisms. In the presence of CFRP strip, the final failure 
comes with the debonding of the strip from the TRC surface or by the delamination between successive layers of the TRC at the bottom (see Fig. 4c and d). The debonding of the strip does not physically exist in the hybrid beams without CFRP while they fracture with matrix cracking at the bottom due to limited resistance to the high tensile stresses developed. These differences are well depicted in the AE parameters behaviour.

For the analysis, the AE activity was divided to stages according to the load (from low, stage 1 to high, stage 10). The average values of the AE parameters AF and RA for each stage are shown in Fig. 5. Focusing on the beams without CFRP, one notices that their activity in terms of this plot is contained in small windows, without much fluctuations as the load increases. AF is typically close to $100 \mathrm{kHz}$ or higher and RA value is limited below $3000 \mu \mathrm{s} / \mathrm{V}$. The situation changes a lot when the beams with CFRP are considered. There, the points are pushed to the lower right part of the graph even from the first stage. This shows that the fracture behavior of the CFRP reinforced beams has different characteristics from the start, indicating that shear stresses are active and will eventually lead to the final failure, while cracking becomes less evident. Furthermore, as load increases for the later stages there is a drop of AF and increase of RA until the final stage (10) which is the last point for both beams just before collapse. This indicates that in the CFRP reinforced beams, the shear mechanisms continuously gain in importance as the material is progressively loaded to failure. The comparison of the two types of behavior shows the benefit of using simple AE parameters for monitoring and even predicting the final failure mode. It is mentioned that a great support for the $\mathrm{AE}$ conclusions, came in this study by the concurrent use of Digital Image Correlation (DIC), which delivered the surface strain map in relation to the applied load. The moments when the surface strain is suddenly decreased, are indicative of delamination events. This allows to safely assigning the AE activity recorded at these moments with the correct fracture mechanism limiting the assumptions that sometimes are inevitable in $\mathrm{AE}$ characterization [8].

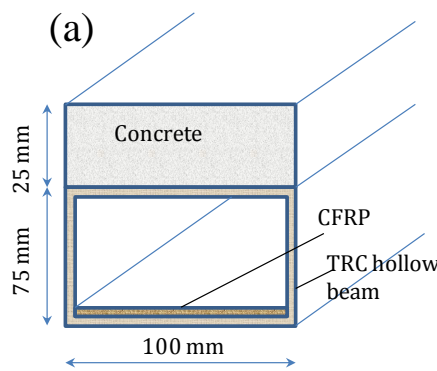

(b)

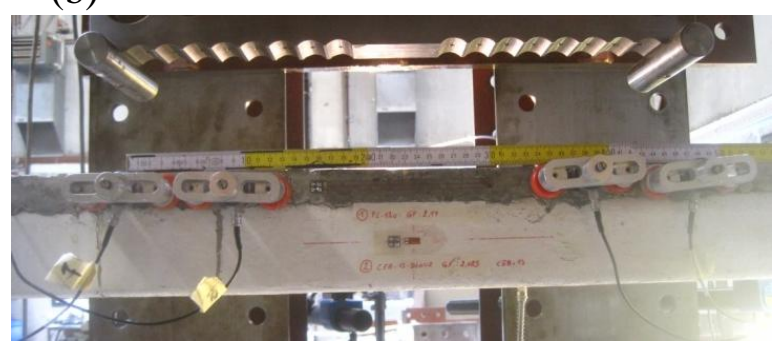

(c)

(d)

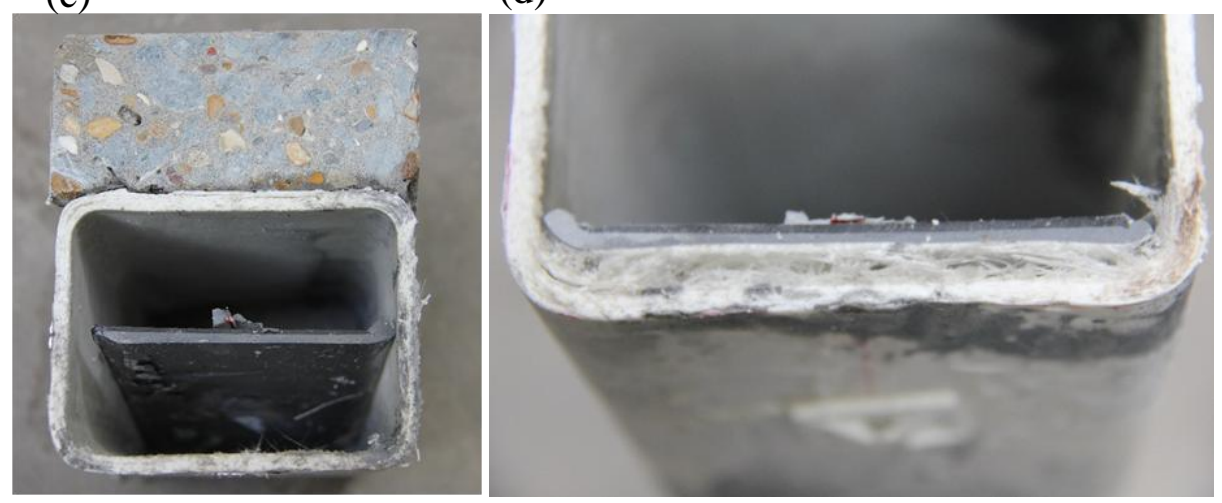

Fig. 4 (a) A typical cross section of the hybrid beams. (b) A photograph before the test. Four AE sensors are seen on the top concrete layer, covering a central distance of $460 \mathrm{~mm}$. On top of the sensors the magnetic clamps are visible. (c) Debonding between the internal CFRP and TRC, (d) delaminations between successive layers of TRC. 


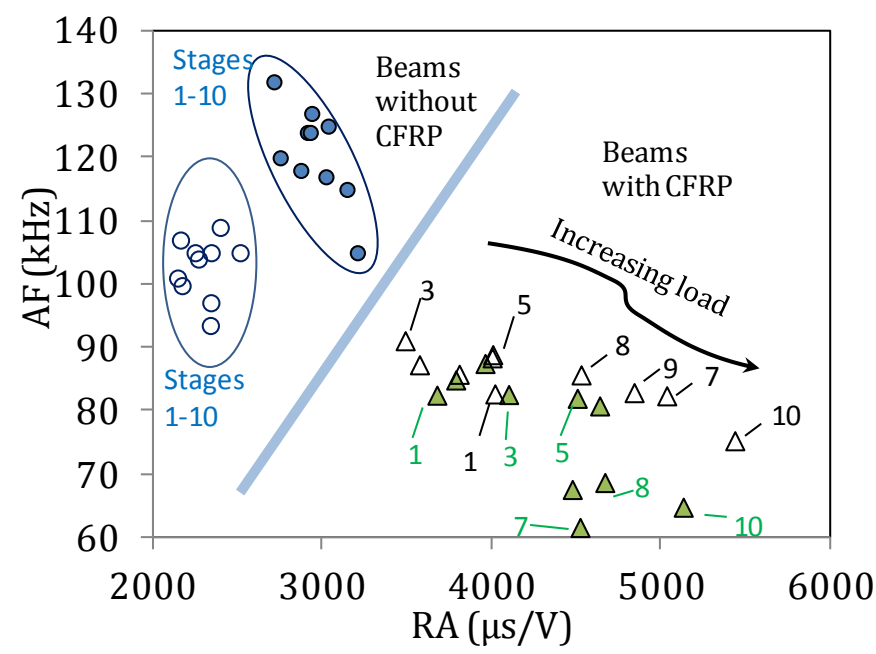

Fig. 5 AF vs. RA plot for different loading stages (low=1, maximum=10) for four hybrid beams.

\section{TESTING OF GRANITE}

Mechanical behavior of granite is of interest in different fields, from restoration of cultural heritage monuments to underground engineering and excavation works $[9,10]$. AE has been used for monitoring the fracture and localizing the sources. In most cases in literature the load is compressive and leads to all different fracture events (tensile, shear and mixed-mode) [11,12]. In this case two modes were isolated. The first is the simple bending mode through the 3-point bending test. Then with a modification on this test (adjustment of the supports and introduction of a notch at one side) the shear mode was targeted. As FEM simulations show this modified test is not a pure shear test but mixed mode with the shear components much stronger than the normal [13]. The geometry and snapshot of the modified experiment are seen in Fig. 6a, while fractured specimens from the "shear" and the bending tests are seen in Fig. $6 \mathrm{~b}$ and c respectively.

(a)

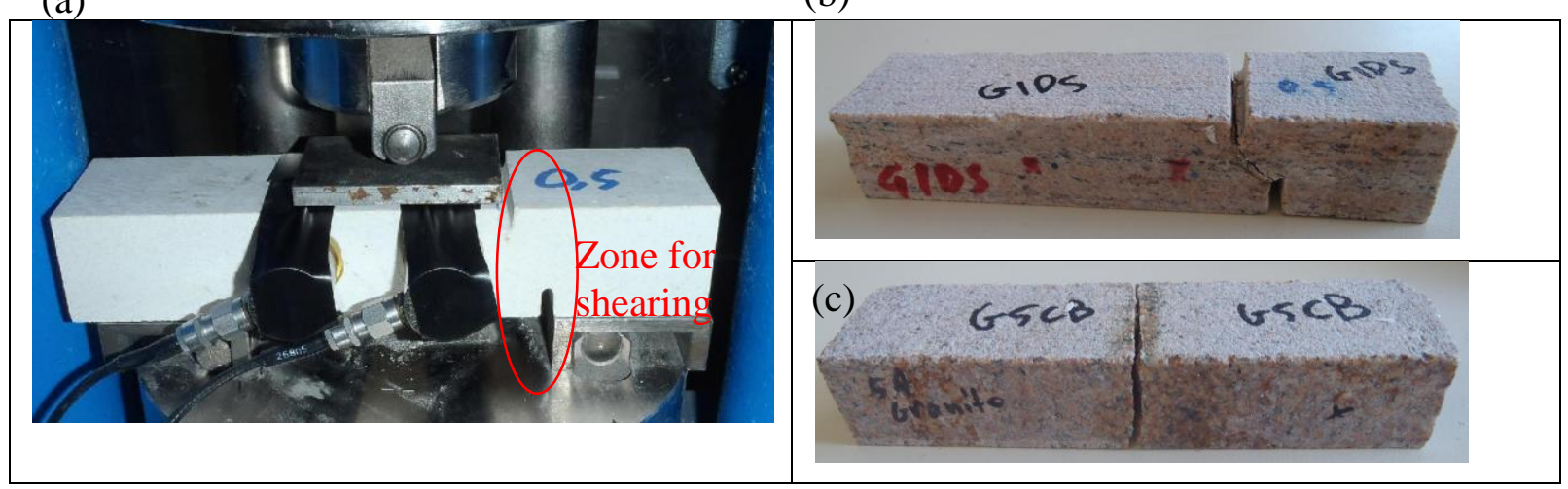

Fig. 6 (a) Modified bending test to promote shear. Fractured specimen of the modified setup (b) and of normal 3p-bending test (c).

Again the AE activity was divided in stages according to load; in this case two stages were defined containg AE recorded after the start of the test at low load and just before final failure. The $\mathrm{AE}$ received at these two steps was compared for their parameters. Fig. 7a shows the distribution of the RT of the received signals for the $1^{\text {st }}$ stage of low load, while Fig. $7 \mathrm{~b}$ includes the corresponding distribution for the higher load stage $\left(2^{\text {nd }}\right)$. It is seen that the RT parameter of AE during the early loading is very much influenced by the macroscopical stress field. The modified "shear-like" testing results in much higher RT and longer signals in general. Apart from the difference in the peak of the distributions ( $20 \mu \mathrm{s}$ for bending and 60 for shear), considering the whole distribution of hits with RT higher than $200 \mu$ s that are not shown in the graph, the bending setup resulted in an average of $45 \mu \mathrm{s}$ while the shear in $211 \mu \mathrm{s}$. This shows the sensitivity of RT on the applied stress field (bending with strong tensile component vs. mixed with stronger shear). This behavior was repeatable to three specimens for each test and for another type of granite [14]. An important detail is that at the last 
stage of loading the differences are minimized between the two modes of fracture, as shown in Fig. $7 \mathrm{~b}$. The peak of RT distribution is nearly the same for bending and shear while the averages are much closer. This is in accordance to literature that states that after the fracture is initiated and close to final failure, it follows a pattern "independent of the actual failure mechanism" and the energy dissipation is "a surface-dominated phenomenon, analogously to the tensile behavior" [11]. Therefore, it comes in agreement with the fact that most of the differences, are seen in the early behavior, while later failure (even if it comes from the modified "shear" test) resembles tensile behavior, and thus exhibits lower RT values during high load.
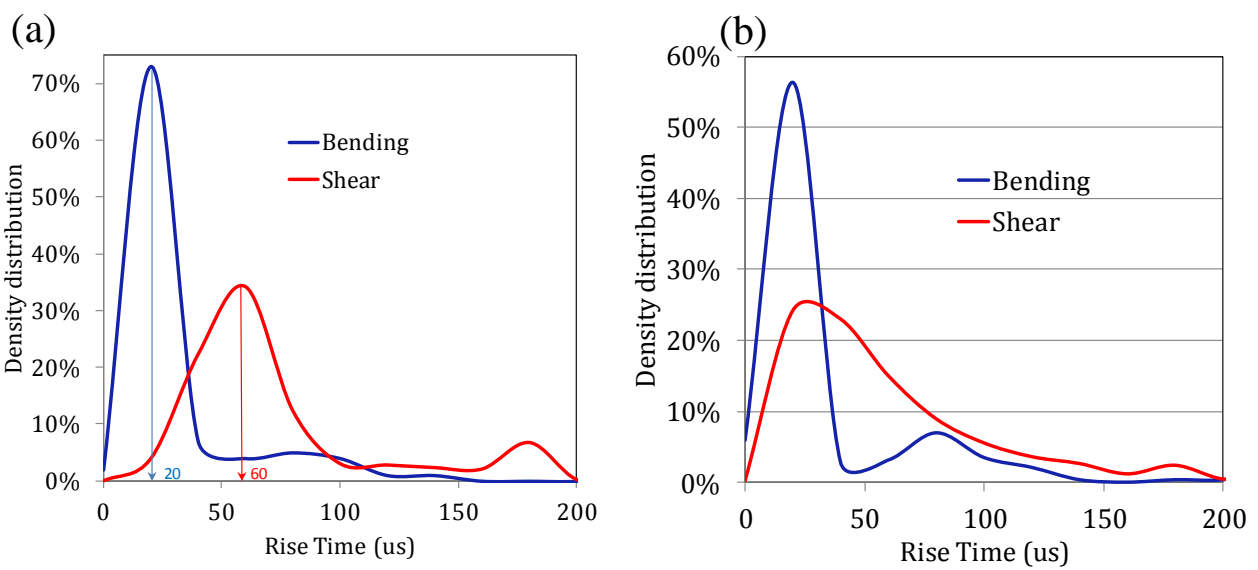

Fig. 7 RT distributions for bending and "shear" tests (a) at low load, (b) high load

\section{CRACK PROPAGATION IN METALS FROM 3D-PRINTING}

The next example concerns the crack propagation in additive manufacturing (AM) metal components, or else 3D-printed. The specific research targets the fracture behaviour of such systems in order to be used as Structural Health Monitoring (SHM) devices embedded in large structures. The important detail is a capillary tube that is designed and printed within the metal sensor. This capillary carries an internal pressure lower than the atmospheric. When a crack propagates into the sensor and breaks the capillary tube, the pressure will be equalized, something that is measured by a pressure sensor [15]. This is a way to check for crack nucleation in the structure and schedule any maintenance or replacement actions. To do so the fracture properties of the system should be studied in order to ensure that the capillary does not deteriorate the life span of the structure (e.g. by stress concentration, weakening of the cross-section). These systems (in their simple testing form beams of rectangular cross section with a capillary along the longitudinal axis) were tested in $4 \mathrm{p}$-bending with concurrent monitoring of $\mathrm{AE}$. This was a good opportunity to check the $\mathrm{AE}$ behaviour of this innovative material which has not been monitored before. Briefly, the specimens presented herein were AISI 316L produced by laser metal deposition (LMD). LMD is a process that uses the energy of a laser beam to melt and bind together metal powder particles. This procedure involves manufacturing layer by layer while residual stresses are formed within the material, necessitating studies of their mechanical and acoustic behaviour, as they cannot be a priori considered identical to traditional metals. Their geometry can be seen in Fig. 8a. The embedded capillary has a diameter of 3 $\mathrm{mm}$ and the specimens were fatigued under maximum load $34 \mathrm{kN}$ until the pressure sensor measured an increase in internal pressure indicating the presence of a crack. The opening of the crack is very small and certainly not visible; thus further tests should be conducted to confirm the presence and the location of the crack. X-rays, eddy currents and liquid penetrants were also used [16]. AE monitoring was conducted during subsequent static loading in a stepwise manner until their previous maximum load of $34 \mathrm{kN}$ that the crack was detected by the pressure transducer. 

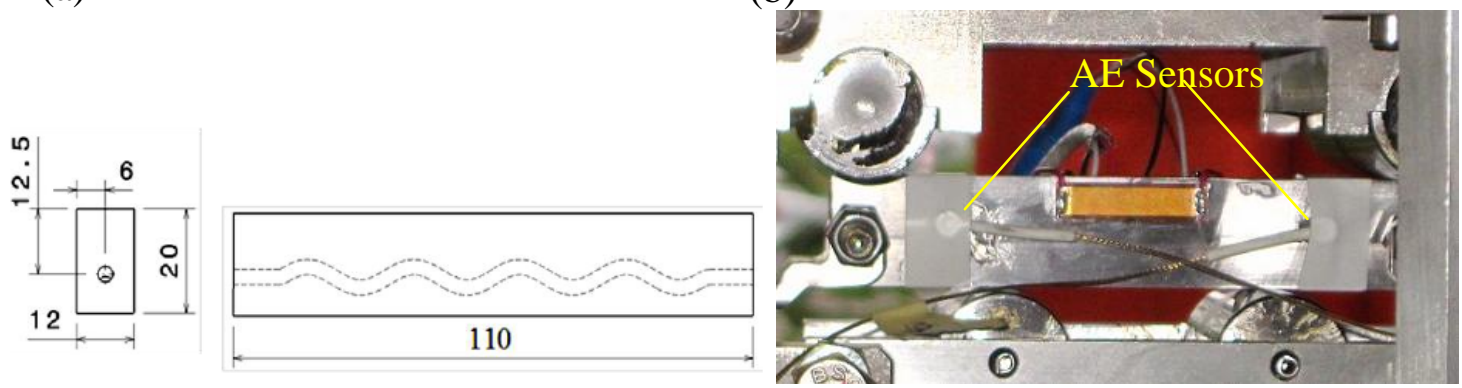

Fig. 8 (a) Geometry of the 3D-printed specimen and (b) photograph during testing.

The acoustic emission sensors were attached between the supports with a separation of $80 \mathrm{~mm}$ (Fig. $8 \mathrm{~b}$ ). The sensors had a broadband response and peak frequency at $450 \mathrm{kHz}$. More details can be found in [16]. Fig. 9 shows the event cumulative number along with load. Initially the rate is moderate until the load reaches close to the previous maximum of $34 \mathrm{kN}$ at which the crack was detected by the "eSHM" system [16], a behaviour related to the well known "Kaiser effect" in AE. It is characteristic that the cumulative number of events is seven until $32 \mathrm{kN}$ and, up to $34 \mathrm{kN}$, the population has already increased to 21. Similar conclusions can be drawn from Figure 9b, where the amplitude and the load history vs. time are shown for the same specimen. Up to the load of $32 \mathrm{kN}$ events do not exceed the amplitude of $50 \mathrm{~dB}$. However, after that point there is also a population of signals above that level up to $75 \mathrm{~dB}$. It is clearly observed that the $\mathrm{AE}$ hits with the highest amplitudes appear at the moment when the load is close to the maximum value of the load when the crack was detected (between 30 and $34 \mathrm{kN}$ ), indicating crack propagation.

(a)

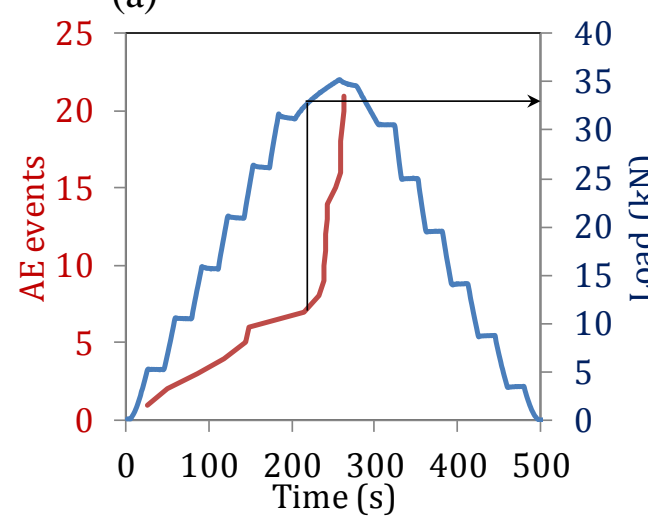

(b)

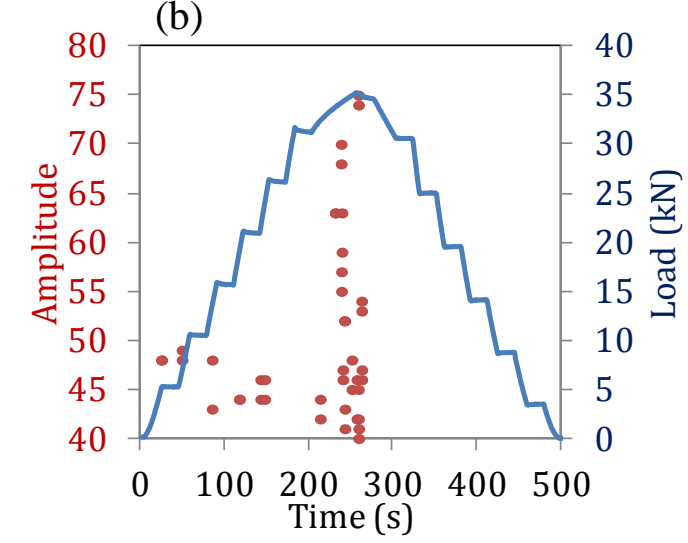

Fig. 9. (a) AE events and (b) AE amplitude along with load history for the 3D printed metal under bending.

Despite the small dimensions and the "ringing" behaviour of the specimen (due to limited attenuation and multiple reflections) and the sensor, linear localization was able to characterize the position of the cracks in these specimens. The events are localized in a range between 56 and $70 \mathrm{~mm}$, being around the actual crack location at $65 \mathrm{~mm}$, as seen in Fig. 10. Although the events are not all located in the same exact position, this distribution is considered quite successful for such a small gauge length $(80 \mathrm{~mm})$ and the unfavourable "noisy" conditions. It is certain that in longer specimens the accuracy of the localization will increase and the whole study suggests that AE can be a valuable tool for monitoring and locating the fracture points in similar SHM 3D-printed components while preliminary results have been published under fatigue loading [17]. 


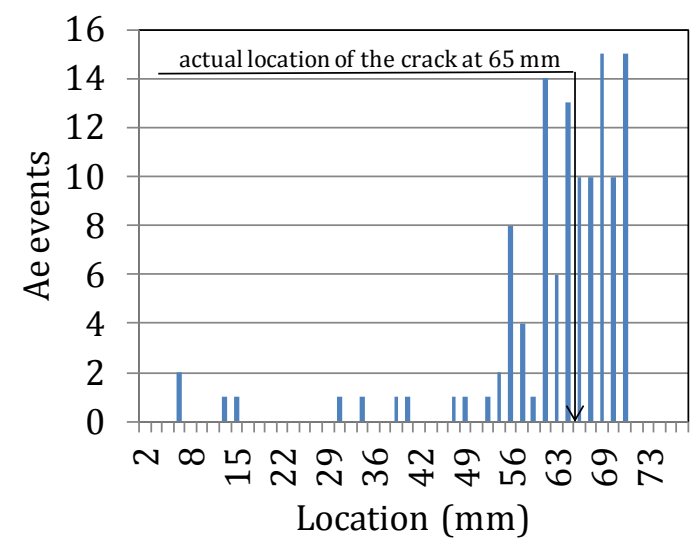

Fig. 10 Distribution of AE location relatively to their linear location in the AM specimen.

\section{CRACKING OF HUMAN BONES}

Bones are very heterogeneous and this complicates the understanding of their fracture behaviour as well as wave propagation conditions. In this case, AE was applied during monotonic loading on human femurs. This is the longest bone of the human body and connects the tibia to the pelvic bone (see Fig. 11a). Injury of this bone (typically near the "head") is common especially for elderly people and is considered one of the main reasons for loss of life quality and life itself. Medical doctors are very interested in the mechanical capacity of the tissue, at which load cracking starts, as well as in the way that the final failure is developed. From the AE point of view there are some challenges, like the interpretation of the data and the geometry of the bone that makes sensor attachment not an easy task [18]. Therefore, in this case application of AE along with observation of the fracture surfaces was a good opportunity to check if $\mathrm{AE}$ parameters that are useful in engineering materials prove similarly powerful to characterize the fracture mode in bone. The experimental setup imposes a combined load of bending on the head and torsion on the main body (diaphysis). The load is applied on the top of the head, while the bone is cast into concrete leaving $120 \mathrm{~mm}$ between the fix point and the point load. A bolt supports the bone at the "trochanter" in order to avoid fracture at the fix point as cantilever while this support, combined with the vertical load creates the torque on the diaphysis (Fig. 11b). Two broadband sensors with peak at $450 \mathrm{kHz}$ were applied on the specimen; one near the fix point and the other on the femur head, as shown in Fig. 11b. Typically the AE activity amassed to cumulative numbers of nearly one thousand hits per specimen and for most of the specimens, the onset of $\mathrm{AE}$ was noticed at approximately $14 \%$ of the maximum load which is the manifestation of the early micro-cracking [19]. A number of the specimens failed at the head due to the high tensile stresses at the top side and others failed by a diagonal crack through the diaphysis in the volume where torsion is expected to be strong. Fig. 12 shows typical cases of the different fracture modes. In Fig. 12a an example of failure near the head is included, while Fig. 12b shows a case of diagonal crack through the body of the bone.

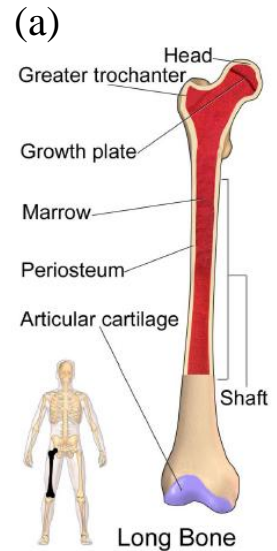

(b)

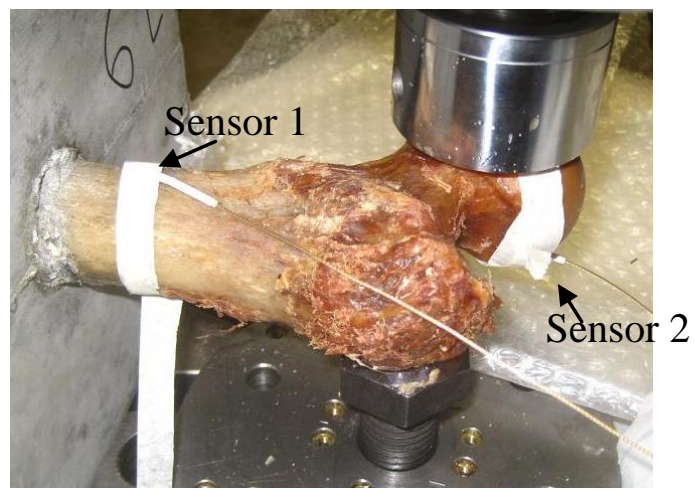

Fig. 11 (a) Human femur bone, [20] (b) test setup showing also two AE sensors. 


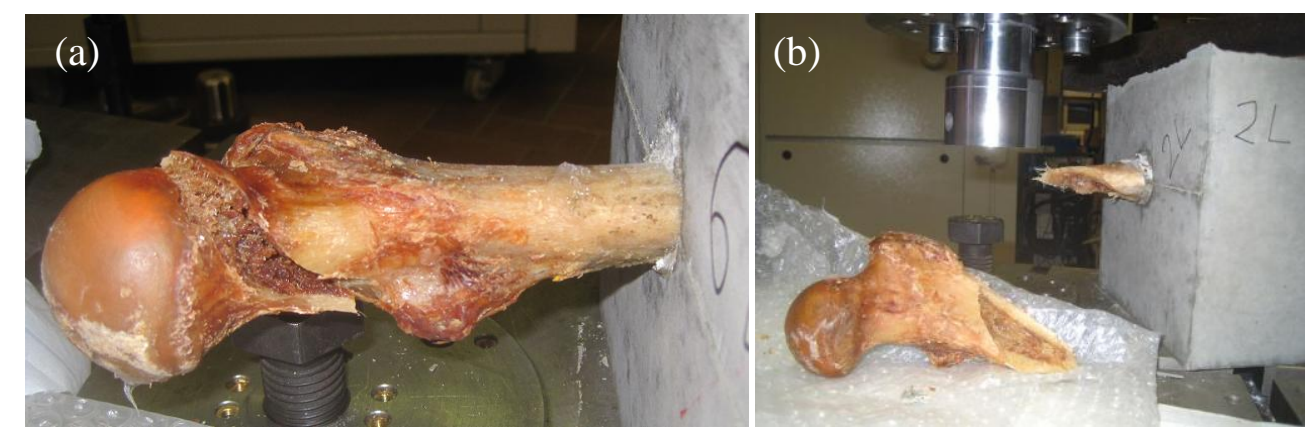

Fig. 12 (a) Bending failure at the head, (b) torsion failure through the diaphysis.

Results of AE are very encouraging despite the large experimental scatter that is inevitable due to heterogeneity, different dimensions of the specimens and slightly different sensor location points. For specimens fractured near the fix point, both sensors registered average value of RT higher than $30 \mu \mathrm{s}$, $(31.5 \mu \mathrm{s}$ to $82.5 \mu \mathrm{s})$ while for specimens broken at the head the average value was always lower than $30 \mu \mathrm{s}(3.6 \mu \mathrm{s}$ to $28.4 \mu \mathrm{s})$. In a previous study of combined bending and torsion load in femur bones RT has been used to distinguish between nucleation or propagation of cracking and friction of the existing crack sides [21]. In similar fashion the RA value for dominant torsion cracking was between $1745 \mu \mathrm{s} / \mathrm{V}$ and $14544 \mu \mathrm{s} / \mathrm{V}$, while for bending fracture of the head it varied between $720 \mu \mathrm{s} / \mathrm{V}$ and $2397 \mu \mathrm{s} / \mathrm{V}$ exhibiting limited overlap. It is characteristic that for the torsion cracking more than $18 \%$ of the hits exhibited RA higher than $5 \mathrm{~ms} / \mathrm{V}$, while for the bending fracture of the head the corresponding percentage was only $2.7 \%$ (see Fig. 13). This is compatible with the experience from engineering materials, where the shear mode of cracks emits longer AE waveforms with higher rise time than tensile cracks $[8,14]$. The major benefit of such study is to provide more insight in the basic understanding of the fracture of the bone as use for fracture diagnostic purposes is not straightforward. However, it is stated that $\mathrm{AE}$ has been used under suitable conditions for the clinical characterization of patients with osteoarthritis [22].
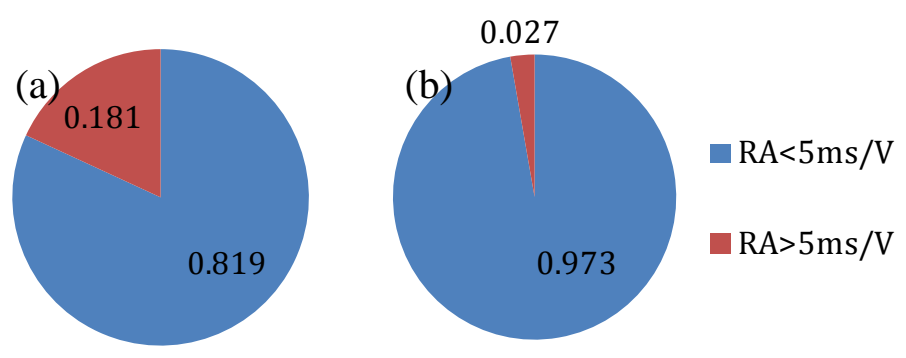

Fig. 13 Percentage of AE hits population relatively to their RA value for (a) torsion cracking of the femur diaphysis and (b) bending cracking of the head, as recorded by the sensor on the head.

\section{PULL-OUT OF REINFORCING BARS FROM CONCRETE}

The last case concerns the monitoring of pull-out experiment from concrete matrix. This test is conducted mainly to examine the adhesion between the rebar and the matrix. The external force applied on the bar is transferred to the matrix through shear stresses at the interface. The way the material behaves and fractures depends on the bond and the possible mechanical interlocking points [23,24]. In case the bonding is low, the bar is easily moved out of the matrix without resistance as it slips along the interface. On the other hand in the presence of good bonding, the stresses are transferred into the matrix and cracks are deflected from the rebar-concrete interface into the concrete matrix. In the present case, numerous specimens were produced having as variable the moment after mixing of concrete when the rebar was embedded into the matrix. The steel rebars had a diameter of $16 \mathrm{~mm}$ and their embedded length in concrete was $100 \mathrm{~mm}$. More details in [25]. The results showed that there were three basic fracture modes. One was the plain pull-out without visible concrete cracking (Fig. 14a). Another was the case that apart from the pull-out, visible extensive cracking was developed into the concrete specimen (Fig. 14b). There was also a number of specimens with limited cracking on the matrix. In this manuscript, the focus is given on the AE behavior so we compare the two extreme cases; the AE activity of the pull-out group of specimens and that of the cracking group. 


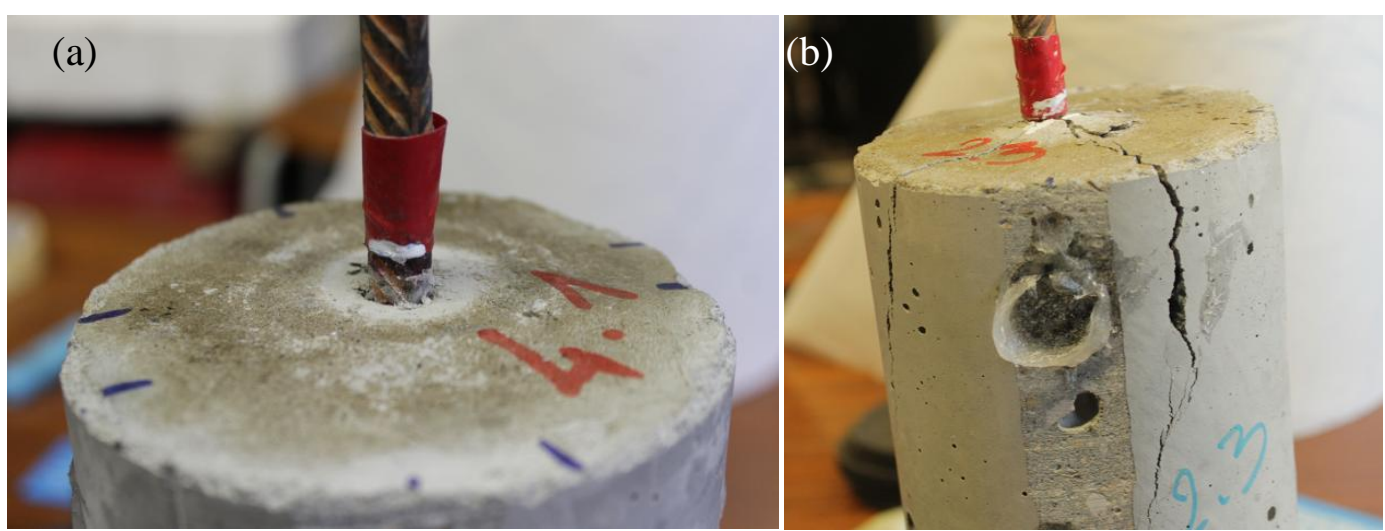

Fig. 14 (a) Pure pull-out, (b) pull-out with cracking of the concrete matrix.

Fig. 15a shows a photo of the AE setup and Fig. 15b shows the history of duration of AE signals for two indicative cases. The line is the moving average of the recent 200 hits. Both lines start from a low value of approximately $500 \mu \mathrm{s}$. However, for the pure pull-out specimen a steep increase is evidenced reaching the level of $3000 \mu \mathrm{s}$. At the same time the line of the specimens that cracked is constantly lower and only a small peak up to $1000 \mu$ s is observed. In average, the pure pull-out specimens exhibited an AE duration of $1023 \mu \mathrm{s}$, while the cracked specimens $418 \mu \mathrm{s}$. This large difference signifies a difference in the fracture mechanisms and corresponds well with the known trends from other loading scenaria; shear like fractures emit longer signals compared to the tensile events. In the present case, when only pull-out is exhibited, the AE events reasonably come from the shearing and the friction between the rebar and concrete. On the other hand specimens that sustain global damage (Fig. 14b) emit signals from matrix cracking as well, which has been shown to contribute shorter signals as seen in other cases in this manuscript and in literature [5,14]. Systematic differences are evidenced in other parameters like AF, though not so clear. The specimens of the pure pull out mode exhibit an average AF of $76 \mathrm{kHz}$ while the "cracking" mode emits an average of 92 $\mathrm{kHz}$. If the activity is separated in stages, the activity after peak is quite indicative. The "pull-out" specimens exhibit durations of more than $1200 \mu$ s and frequencies of $71 \mathrm{kHz}$ in average, while the cracked specimens have a duration of $500 \mu$ s and frequency around $90 \mathrm{kHz}$ meaning that for the latter the fracture mechanisms leading to the emissions is not just shearing friction but includes cracking incidents as well which continue even after the peak load point.
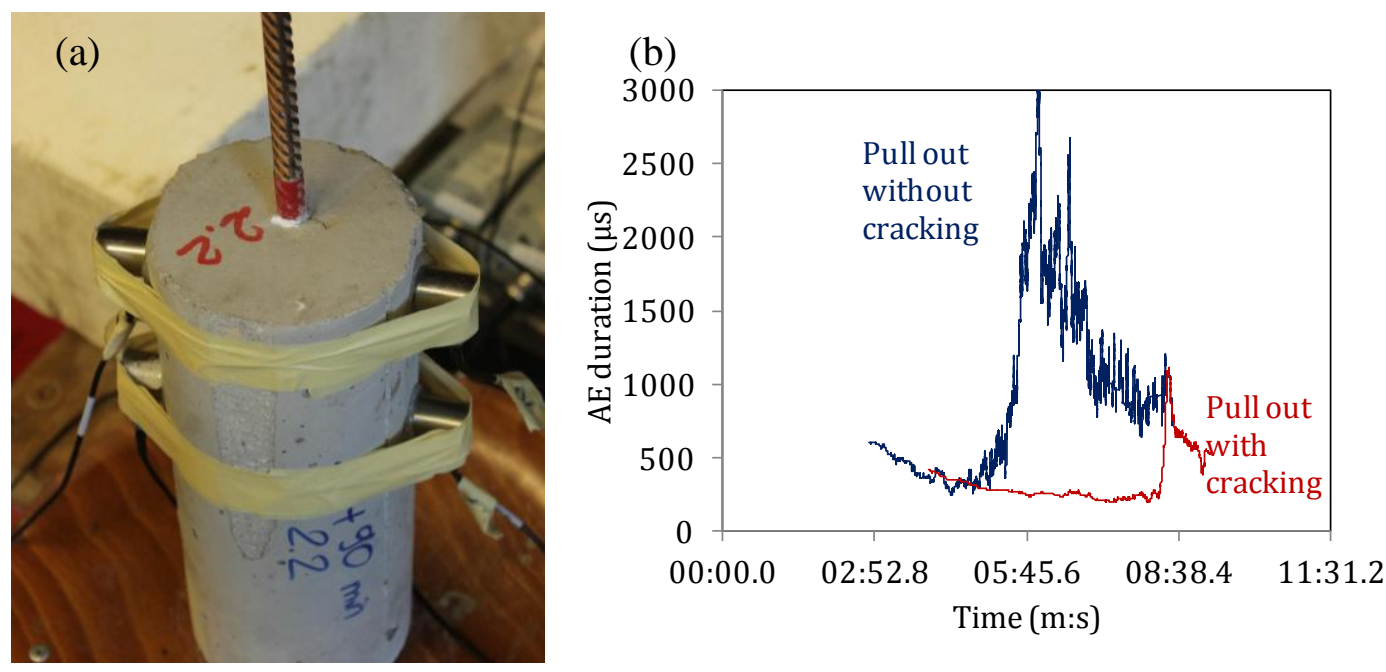

Fig. 15 (a) Concrete specimen with rebar and four AE sensors before loading, (b)AE duration for two concrete specimens in the rebar pull-out test.

\section{CONCLUSIONS}

The present paper discusses several recent cases of fracture monitoring with $\mathrm{AE}$ studied in the department of Mechanics of Materials and Constructions of the Vrije Universiteit Brussel. The focus is in characterization of the mode of failure a field that is currently opening worldwide and concentrates efforts from several research groups worldwide. In the few indicative above mentioned 
cases it is examined if simple $\mathrm{AE}$ waveform parameters are sensitive and can provide real time information for the fracture mode in different materials. These parameters mainly include the average frequency (AF), RA value and rise time (RT), the suitability of which is demonstrated. The reason is due to the motion of the crack sides that emit elastic energy in different wave modes. Shear mechanisms that include movement of the planes in parallel but opposite directions result in longer signals of lower frequency than tensile which imposes movement vertical to the crack planes. However, it should always be kept in mind that specific values should not easily be connected with fracture mechanisms. Propagation distance, sensor characteristics etc. may well influence the final values. The importance lies in observing the trends within each experiment, when a change may indicate a shift of mode, e.g. the end of cracking and start of the delaminations in TRC composites. Comparison can also be conducted in different experiments for exactly the same experimental conditions, like in the case of granite specimens under bending or shear. Other techniques that can be used in parallel, give insight and verify the conclusions, like the application of DIC which confirms the change in the strain field and allows to distinguish delaminations and assign the AE recorded at that time to the correct failure mechanism. It is believed that AE characterization should be combined with numerical simulation of wave propagation especially in complicated geometries in order to be able to exclude the influence of geometry (reflections etc.) and make the parameter values less dependent on the conditions. Combination of $\mathrm{AE}$ with numerical simulations and other monitoring techniques is expected to improve the interpretation of the $\mathrm{AE}$ data and increase the reliability of $\mathrm{AE}$ testing.

\section{REFERENCES}

[1] Grosse CU, Ohtsu M. Acoustic Emission Testing. Heidelberg: Springer; 2008.

[2] Christian U. Grosse, Lindsay M. Linzer, Chapter 5: Signal-Based AE Analysis, in: Grosse CU, Ohtsu M. Acoustic Emission Testing. Heidelberg: Springer; 2008.

[3] Tomoki Shiotani, Chapter 4: Parameter Analysis, in: Grosse CU, Ohtsu M. Acoustic Emission Testing. Heidelberg: Springer; 2008.

[4] Verbruggen, S., Tysmans, T., Wastiels, J.: TRC or CFRP strengthening for reinforced concrete beams: an experimental study of the cracking behaviour. Eng. Struct. 77, 49-56 (2014)

[5] Blom, J., El Kadi, M., Wastiels, J., Aggelis, D. G. 2014. Bending fracture of textile reinforced cement laminates monitored by acoustic emission: influence of aspect ratio. Construction and Building Materials, 70:370-378.

[6] De Sutter, S., Tysmans, T., Wozniak, M.: Analytical modelling and experimental testing of hybrid composite-concrete beams in a lightweight floor system. In: ElHatcha, R. (ed.) Proceeding of the 7th International Conference on FRP Composites in Civil Engineering (CICE2014), Vancouver, Canada, August 2014, ISBN 529 9781-77136-308-2

[7] S. Verbruggen, S. De Sutter, S. Iliopoulos, D. G. Aggelis, T. Tysmans Experimental Structural Analysis of Hybrid Composite-Concrete Beams by Digital Image Correlation (DIC) and Acoustic Emission (AE), Journal of Nondestructive Evaluation, DOI 10.1007/s10921-015-0321-9

[8] D.G. Aggelis, S. Verbruggen, E. Tsangouri, T. Tysmans, D. Van Hemelrijck Characterization of mechanical performance of concrete beams with external reinforcement by acoustic emission and digital image correlation, Construction and Building Materials 47 (2013) 1037-1045

[9] A. Moropoulou, K. Polikreti, V. Ruf, G. Deodatis, San Francisco Monastery, Quito, Equador: characterisation of building materials, damage assessment and conservation considerations, Journal of Cultural Heritage 4 (2003) 101-108.

[10] L. Chen, J.F. Liu, C.P. Wang, J. Liu,R. Su, J. Wang, Characterization of damage evolution in granite under compressive stress condition and its effect on permeability, International Journal of Rock Mechanics \& Mining Sciences 71 (2014) 340-349. 
[11] A. Carpinteri, M. Corrado, G. Lacidogna, Heterogeneous materials in compression: Correlations between absorbed, released and acoustic emission energies, Engineering Failure Analysis 33 (2013) 236-250.

[12] L. Jian-po, L. Yuan-hui, X. Shi-da, X. Shuai, J. Chang-yu, Cracking mechanisms in granite rocks subjected to uniaxial compression by moment tensor analysis of acoustic emission, Theoretical and Applied Fracture Mechanics 75 (2015) $151-159$.

[13] D.G. Aggelis, A.C. Mpalaskas, T.E. Matikas, Investigation of different fracture modes in cement-based materials by acoustic emission, Cement and Concrete Research 48 (2013) 1-8.

[14] A.C. Mpalaskas, T. E. Matikas, D. Van Hemelrijck, G.S. Papakitsos, D. G. Aggelis, Acoustic emission monitoring of granite under bending and shear loading, Archives of Civil and Mechanical Engineering, 10.1016/j.acme.2016.01.006

[15] Strantza, M.; De Baere, D.; Rombouts, M.; Maes, G.; Guillaume, P.; Van Hemelrijck, D. Feasibility study on integrated structural health monitoring system produced by metal three-dimensional printing. Struct. Health Monit. 2015, doi:10.1177/1475921715604389.

[16] M. Strantza, D. G. Aggelis, D. de Baere, P. Guillaume and D. van Hemelrijck, Evaluation of SHM System Produced by Additive Manufacturing via Acoustic Emission and Other NDT Methods, Sensors 2015, 15, 26709-26725; doi:10.3390/s 151026709

[17] Strantza, M., Van Hemelrijck, D., Aggelis, D.G., De Baere, D., Guillaume, P. Acoustic emission monitoring of crack propagation in titanium samples 2015 Structural Health Monitoring 2015: System Reliability for Verification and Implementation - Proceedings of the 10th International Workshop on Structural Health Monitoring, IWSHM 2015, Volume 2, 1485-1492.

[18] Shrivastava, S.; Prakash, R. Assessment of bone condition by acoustic emission technique: A review. J. Biomed. Sci. Eng. 2009, 2, 144-154.

[19] Aggelis, D. G., Strantza, M., Louis, O., Boulpaep, F., Polyzos, D., Van Hemelrijck, D., 2015. Fracture of Human Femur Tissue Monitored by Acoustic Emission Sensors, Sensors 15(3):5803-5819.

[20] https://en.wikipedia.org/wiki/Femur\#/media/File:Long_Bone_(Femur).png

[21] Schwalbe, H.-J.; Bamfaste, G.; Franke, R.-P. Non-destructive and noninvasive observation of friction and wear of human joints and of fracture initiation by acoustic emission. Proc. Inst. Mech. Eng. 1999, 213, 41-48.

[22] Shark, L.-K.; Chen, H.; Goodacre, J. Acoustic emission: A potential biomarker for quantitative assessment of joint ageing and degeneration. Med. Eng. Phys. 2011, 33, 534-545.

[23] Hadi, M. N, Bond of high strength concrete with high strength reinforcing steel, Open Civil Engineering Journal, 2, (2008): 143-147

[24] C. U. Grosse, H. Reinhardt, T. Dahm, Localization and classification of fracture types in concrete with quantitative acoustic emission measurement techniques, NDT E Int. 30 (4) (1997) 223-230.

[25] Phuoc Luong Nguyen, J. Vantomme, D. G. Aggelis, Acoustic emission monitoring of reinforcing bars pull-out from concrete matrix, accepted for presentation in the $19^{\text {th }}$ World Conference in Non-Destructive Testing 2016, Munich, Germany, 13-17 June 2016. 